\title{
A Hilbert-type operator with a symmetric homogeneous kernel of two parameters and its applications
}

Keomkyo Seo*

"Correspondence:

kseo@sookmyung.ac.kr Department of Mathematics, Sookmyung Women's University, Hyochangwongil 52, Yongsan-ku, Seoul, 140-742, Korea

\begin{abstract}
We introduce a general homogeneous kernel whose degree is given by two parameters to establish the equivalent inequalities with the norm of a new Hilbert-type operator. As applications, we provide new extended Hilbert-type inequalities with the best possible constant factors.
\end{abstract}

MSC: $47 \mathrm{~A} 07 ; 26 \mathrm{D} 15$

Keywords: Hilbert-type operator; Hilbert-type inequality; beta function; kernel; norm

\section{Introduction}

Let $\left\{a_{n}\right\}$ and $\left\{b_{m}\right\}$ be two sequences of nonnegative real numbers. The well-known Hilbert's inequality says that if $p>1, \frac{1}{p}+\frac{1}{q}=1,0<\sum_{m=1}^{\infty} a_{m}^{p}<\infty$ and $0<\sum_{n=1}^{\infty} b_{n}^{q}<\infty$, then

$$
\sum_{m=1}^{\infty} \sum_{n=1}^{\infty} \frac{a_{m} b_{n}}{m+n}<\frac{\pi}{\sin \left(\frac{\pi}{p}\right)}\left(\sum_{m=1}^{\infty} a_{m}^{p}\right)^{1 / p}\left(\sum_{n=1}^{\infty} b_{n}^{q}\right)^{1 / q}
$$

where the constant factor $\frac{\pi}{\sin \left(\frac{\pi}{p}\right)}$ is the best possible [1]. This inequality has been generalized in numerous ways with introducing suitable parameters and weight coefficients. (For example, see [2-13] and the references therein.) In particular, by introducing a Hilbert-type linear operator with a symmetric homogeneous kernel, one can obtain various Hilbert-type inequalities with the best constant factors. For this purpose, let $k(x, y)$ be a nonnegative symmetric function defined on $(0, \infty) \times(0, \infty)$, i.e., $k(x, y)=k(y, x)$. For $p>1$ and $\frac{1}{p}+\frac{1}{q}=1$, let $\ell^{r}(r=p, q)$ be two normed spaces. If $T$ is a bounded self-adjoint semi-positive definite operator defined by

$$
(T a)(n):=\sum_{m=1}^{\infty} k(m, n) a_{m}, \quad n \in \mathbb{N}
$$

\section{Springer}

(c) 2015 Seo. This article is distributed under the terms of the Creative Commons Attribution 4.0 International License (http://creativecommons.org/licenses/by/4.0/), which permits unrestricted use, distribution, and reproduction in any medium, provided you give appropriate credit to the original author(s) and the source, provide a link to the Creative Commons license, and indicate if changes were made. 
for $a=\left\{a_{m}\right\}_{m=1}^{\infty} \in \ell^{p}$, or similarly,

$$
(T b)(m):=\sum_{n=1}^{\infty} k(m, n) b_{n}, \quad m \in \mathbb{N}
$$

for $b=\left\{b_{n}\right\}_{n=1}^{\infty} \in \ell^{q}$. The operator $T$ is called the Hilbert-type operator and the function $k(x, y)$ is called the symmetric kernel of $T$. In view of this point, Hilbert's inequality (1) can be expressed by

$$
(T a, b) \leq \frac{\pi}{\sin \left(\frac{\pi}{p}\right)}\|a\|_{p}\|b\|_{q},
$$

where the kernel $k(x, y)=\frac{1}{x+y}$ and the formal inner product $(T a, b)$ between $T a$ and $b$ is given by $(T a, b):=\sum_{n=1}^{\infty}(T a)(n) b_{n}$. Motivated by this observation, Yang [14] defined a Hilbert-type linear operator $T: \ell^{r} \rightarrow \ell^{r}(r=p, q)$ with the kernel $k(x, y)=\frac{(x y)^{\frac{\lambda-1}{2}}}{(x+y)^{\lambda}}$ of degree -1 . As a consequence, he was able to prove that if $p>1, \frac{1}{p}+\frac{1}{q}=1, a_{m}, b_{n} \geq 0$, $1-2 \min \left\{\frac{1}{p}, \frac{1}{q}\right\}<\lambda<1+2 \min \left\{\frac{1}{p}, \frac{1}{q}\right\}$, then the following two inequalities are equivalent:

$$
\begin{aligned}
& \sum_{n=1}^{\infty} \sum_{m=1}^{\infty} \frac{(m n)^{\frac{\lambda-1}{2}} a_{m} b_{n}}{m^{\lambda}+n^{\lambda}}<\frac{1}{\lambda} B\left(\frac{q(\lambda+1)-2}{2 q \lambda}, \frac{p(\lambda+1)-2}{2 p \lambda}\right)\|a\|_{p}\|b\|_{q}, \\
& \left\{\sum_{n=1}^{\infty}\left(\sum_{m=1}^{\infty} \frac{(m n)^{\frac{\lambda-1}{2}} a_{m}}{m^{\lambda}+n^{\lambda}}\right)^{p}\right\}^{\frac{1}{p}}<\frac{1}{\lambda} B\left(\frac{q(\lambda+1)-2}{2 q \lambda}, \frac{p(\lambda+1)-2}{2 p \lambda}\right)\|a\|_{p},
\end{aligned}
$$

where $B(u, v)$ denotes the beta function defined by

$$
B(u, v):=\int_{0}^{\infty} \frac{t^{u-1}}{(1+t)^{u+v}} d t=B(u, v) \quad(u, v>0) .
$$

Moreover, the constant factor $\frac{1}{\lambda} B\left(\frac{q(\lambda+1)-2}{2 q \lambda}, \frac{p(\lambda+1)-2}{2 p \lambda}\right)$ is the best possible. In 2010, Jin and Debnath [15] generalized the Hilbert-type linear operator whose kernel is symmetric and homogeneous of degree -1 . In fact, they obtained several extended Hilbert-type inequalities by using the kernel $k(x, y)=\frac{1}{\left(x^{\frac{1}{\lambda}}+y^{\frac{1}{\lambda}}\right)^{\lambda}}(\lambda>0)$. For instance, they proved that if $p>1$, $\frac{1}{p}+\frac{1}{q}=1, \alpha, \beta>0,0<\lambda \leq \min \left\{\frac{q}{\alpha}, \frac{p}{\beta}\right\}$, then the following two inequalities are equivalent:

$$
\begin{aligned}
& \sum_{n=1}^{\infty} \sum_{m=1}^{\infty} \frac{a_{m} b_{n}}{\left(m^{\alpha}+n^{\beta}\right)^{\lambda}}<\frac{B\left(\frac{\lambda}{p}, \frac{\lambda}{q}\right)}{\alpha^{\frac{1}{q}} \beta^{\frac{1}{p}}}\left(\sum_{m=1}^{\infty} m^{(p-1)(1-\alpha \lambda)}\left|a_{m}\right|^{p}\right)^{\frac{1}{p}}\left(\sum_{n=1}^{\infty} n^{(q-1)(1-\beta \lambda)}\left|b_{n}\right|^{q}\right)^{\frac{1}{q}}, \\
& \left\{\sum_{n=1}^{\infty} n^{\beta \lambda-1}\left(\sum_{m=1}^{\infty} \frac{a_{m}}{\left(m^{\alpha}+n^{\beta}\right)^{\lambda}}\right)^{p}\right\}^{\frac{1}{p}}<\frac{B\left(\frac{\lambda}{p}, \frac{\lambda}{q}\right)}{\alpha^{\frac{1}{q}} \beta^{\frac{1}{p}}}\left(\sum_{m=1}^{\infty} m^{(p-1)(1-\alpha \lambda)}\left|a_{m}\right|^{p}\right)^{\frac{1}{p}},
\end{aligned}
$$

where the constant factor $\frac{B\left(\frac{\lambda}{p}, \frac{\lambda}{q}\right)}{\alpha^{\frac{1}{q}} \beta^{\frac{1}{p}}}$ is the best possible. See [16-23] for other Hilbert-type operators and the corresponding extended Hilbert-type inequalities with the best factors.

Most of the previous results were, however, obtained by using the Hilbert-type operator with the symmetric homogeneous kernel of $-\lambda$-order, which depends on a parameter $\lambda>0$. In this paper, we introduce a more general homogeneous kernel whose degree is 
given by two parameters (Definition 2.3). We establish the equivalent inequalities with the norm of a new Hilbert-type operator (Theorem 3.1). As applications, we provide new extended Hilbert-type inequalities with the best possible constant factors (Corollary 4.1 and Cases 1-3).

\section{Hilbert-type operator with a symmetric homogeneous kernel whose degree is given by two parameters}

For completeness, we begin with the following definitions and notations.

Definition 2.1 Let $p>1, n_{0} \in \mathbb{Z}, w(n) \geq 0\left(n \geq n_{0}, n \in \mathbb{Z}\right)$. Define the normed space $\ell_{w, n_{0}}^{p}$ by

$$
\ell_{w, n_{0}}^{p}:=\left\{a=\left\{a_{n}\right\}_{n=n_{0}}^{\infty}:\|a\|_{p, w}:=\left(\sum_{n=n_{0}}^{\infty} w(n)\left|a_{n}\right|^{p}\right)^{1 / p}<\infty\right\} .
$$

Definition 2.2 Let $\lambda_{1}, \lambda_{2}, \lambda>0$ satisfying that $\lambda=\lambda_{1}+\lambda_{2}$. Denote by $F_{n_{0}}(r)\left(n_{0} \in \mathbb{Z}\right)$ the set of all real-valued $C^{1}$-functions $\phi(x)$ satisfying the following conditions:

(1) $\phi(x)$ is strictly increasing in $\left(n_{0}-1, \infty\right)$ with $\phi\left(\left(n_{0}-1\right)+\right)=0, \phi(\infty)=\infty$.

(2) For $\alpha>0, \frac{\phi^{\prime}(x)}{\phi(x)^{\alpha+1-\lambda_{i}}}$ is decreasing in $\left(n_{0}-1, \infty\right)$.

Let $p>1, \frac{1}{p}+\frac{1}{q}=1, \lambda=\lambda_{1}+\lambda_{2}, \lambda_{1}, \lambda_{2}, \lambda>0$. For $\phi(x) \in F_{m_{0}}(r)$ and $\psi(y) \in F_{n 9}(s), r, s>1$, we define the following weight functions:

$$
\begin{aligned}
& w_{1}(m):=\frac{\phi(m)^{p\left(\alpha+1-\lambda_{2}\right)-1}}{\phi^{\prime}(m)^{p-1}}, \quad w_{2}(n):=\frac{\psi(n)^{q\left(\alpha+1-\lambda_{1}\right)-1}}{\psi^{\prime}(n)^{q-1}}, \\
& \widetilde{w}_{1}(n):=\frac{\psi^{\prime}(n)}{\psi(n)^{p\left(\alpha-\lambda_{1}\right)+1}}, \quad \widetilde{w}_{2}(m):=\frac{\phi^{\prime}(m)}{\phi(m)^{q\left(\alpha-\lambda_{2}\right)+1}} .
\end{aligned}
$$

Definition 2.3 Let $\lambda_{1}, \lambda_{2}, \lambda>0$ satisfying that $\lambda=\lambda_{1}+\lambda_{2}$. For $\alpha>0$ and $x, y>0, K_{\alpha, \lambda}(x, y)$ is a continuous real-valued function on $(0, \infty) \times(0, \infty)$ satisfying the following properties:

(1) $K_{\alpha, \lambda}(x, y)$ is a symmetric homogeneous function of degree $2 \alpha-\lambda$, that is,

$$
\begin{aligned}
& K_{\alpha, \lambda}(x, y)=K_{\alpha, \lambda}(y, x), \\
& K_{\alpha, \lambda}(t x, t y)=t^{2 \alpha-\lambda} K_{\alpha, \lambda}(x, y) \quad \text { for any } t>0 .
\end{aligned}
$$

(2) $K_{\alpha, \lambda}(x, y)$ is decreasing with respect to $x$ and $y$, respectively.

(3) For sufficiently small $\varepsilon \geq 0$, the following integral

$$
\widetilde{K}_{\alpha, \lambda}\left(\lambda_{i}, \varepsilon\right):=\int_{0}^{\infty} K_{\alpha, \lambda}(1, t) t^{-1+\lambda_{i}-\alpha-\varepsilon} d t
$$

exists for $i=1,2$. Moreover, assume that $\widetilde{K}_{\alpha, \lambda}\left(\lambda_{i}, 0\right):=K_{\alpha}\left(\lambda_{i}\right)>0$ and $\widetilde{K}_{\alpha, \lambda}\left(\lambda_{i}, \varepsilon\right)=K_{\alpha}\left(\lambda_{i}\right)+o(1)$ as $\varepsilon \rightarrow 0+$.

(4) Given $p>1, \phi(x) \in F_{m_{0}}(r)$, and $\psi(y) \in F_{n_{0}}(s)(r, s>1)$,

$$
\sum_{n=n_{0}}^{\infty} \frac{\psi^{\prime}(n)}{\psi(n)^{1+\varepsilon}} \int_{0}^{\frac{\phi\left(m_{0}\right)}{\psi(n)}} K_{\alpha, \lambda}(1, t) t^{-1+\lambda_{i}-\alpha-\frac{\varepsilon}{p}} d t=O(1)
$$

as $\varepsilon \rightarrow 0+$. 
Lemma 2.4 Let $\lambda_{1}, \lambda_{2}, \lambda>0$ satisfying that $\lambda=\lambda_{1}+\lambda_{2}$. For any $\alpha>0$, we have

$$
K_{\alpha}\left(\lambda_{1}\right)=K_{\alpha}\left(\lambda_{2}\right)
$$

Proof Since

$$
K_{\alpha}\left(\lambda_{1}\right)=\widetilde{K}_{\alpha, \lambda}\left(\lambda_{1}, 0\right)=\int_{0}^{\infty} K_{\alpha, \lambda}(1, t) t^{-1+\lambda_{1}-\alpha} d t
$$

letting $t=\frac{1}{s}$ gives

$$
K_{\alpha}\left(\lambda_{1}\right)=\int_{0}^{\infty} K_{\alpha, \lambda}(1, s) s^{-1+\lambda_{2}-\alpha} d s=K_{\alpha}\left(\lambda_{2}\right) .
$$

In view of Lemma 2.4, we may assume that

$$
K_{\alpha}(\lambda):=K_{\alpha}\left(\lambda_{1}\right)=K_{\alpha}\left(\lambda_{2}\right)
$$

Lemma 2.5 Let $p>1, \frac{1}{p}+\frac{1}{q}=1, \lambda_{1}+\lambda_{2}=\lambda, \lambda_{1}, \lambda_{2}>0, \alpha>0$. For $\phi(x) \in F_{m_{0}}(r)$ and $\psi(y) \in$ $F_{n_{0}}(s), r, s>1$, define the weight coefficients $W_{1}(m)$ and $W_{2}(n)$ by

$$
\begin{aligned}
& W_{1}(m):=\sum_{n=n_{0}}^{\infty} K_{\alpha, \lambda}(\phi(m), \psi(n)) \frac{\phi(m)^{\lambda_{2}-\alpha}}{\psi(n)^{\alpha+1-\lambda_{1}}} \psi^{\prime}(n), \\
& W_{2}(n):=\sum_{m=m_{0}}^{\infty} K_{\alpha, \lambda}(\phi(m), \psi(n)) \frac{\psi(n)^{\lambda_{1}-\alpha}}{\phi(m)^{\alpha+1-\lambda_{2}}} \phi^{\prime}(m) .
\end{aligned}
$$

Then

$$
W_{1}(m)<K_{\alpha}(\lambda) \text { and } W_{2}(n)<K_{\alpha}(\lambda)
$$

for any $m \geq m_{0}, n \geq n_{0}(m, n \in \mathbb{Z})$.

Proof We have

$$
\begin{aligned}
W_{1}(m) & =\sum_{n=n_{0}}^{\infty} K_{\alpha, \lambda}\left(1, \frac{\psi(n)}{\phi(m)}\right) \frac{\phi(m)^{\alpha-\lambda_{1}}}{\psi(n)^{\alpha+1-\lambda_{1}}} \psi^{\prime}(n) \\
& <\int_{n_{0}-1}^{\infty} K_{\alpha, \lambda}\left(1, \frac{\psi(x)}{\phi(m)}\right) \frac{\psi^{\prime}(x)}{\psi(x)^{\alpha+1-\lambda_{1}}} \phi(m)^{\alpha-\lambda_{1}} d x
\end{aligned}
$$

Setting $t=\frac{\psi(x)}{\phi(m)}$, we get

$$
W_{1}(m)<\int_{0}^{\infty} K_{\alpha, \lambda}(1, t) t^{-1+\lambda_{1}-\alpha} d t=K_{\alpha}(\lambda) .
$$

Similarly, one can obtain $W_{2}(n)<K_{\alpha}(\lambda)$.

Lemma 2.6 Let $p>1, \frac{1}{p}+\frac{1}{q}=1, \lambda_{1}+\lambda_{2}=\lambda, \lambda_{1}, \lambda_{2}>0$. For $a_{m}, b_{n} \geq 0\left(m_{0}, n_{0} \in \mathbb{Z}\right)$, let $a=\left\{a_{m}\right\}_{m=m_{0}}^{\infty} \in \ell_{w_{1}, m_{0}}^{p}$ and $b=\left\{b_{n}\right\}_{n=n_{0}}^{\infty} \in \ell_{w_{2}, n_{0}}^{q}$. Then, for $\phi(x) \in F_{m_{0}}(r)$ and $\psi(y) \in F_{n_{0}}(s)$ 
Geo Journal of Inequalities and Applications <wide>(2015<wide>) 2015:266

Page 5 of 14

$(r, s>1)$, we have

$$
\begin{aligned}
& \left\|\sum_{m=m_{0}}^{\infty} K_{\alpha, \lambda}(\phi(m), \psi(n)) a_{m}\right\|_{p, \widetilde{w}_{1}} \leq K_{\alpha}(\lambda)\|a\|_{p, w_{1}} \text { and } \\
& \left\|\sum_{n=n_{0}}^{\infty} K_{\alpha, \lambda}(\phi(m), \psi(n)) b_{n}\right\|_{q, \widetilde{w}_{2}} \leq K_{\alpha}(\lambda)\|b\|_{q, w_{2}},
\end{aligned}
$$

and hence

$$
\begin{aligned}
& \left\{\sum_{m=m_{0}}^{\infty} K_{\alpha, \lambda}(\phi(m), \psi(n)) a_{m}\right\}_{n=n_{0}}^{\infty} \in \ell_{\tilde{w}_{1}, n_{0}}^{p} \text { and } \\
& \left\{\sum_{n=n_{0}}^{\infty} K_{\alpha, \lambda}(\phi(m), \psi(n)) b_{n}\right\}_{m=m_{0}}^{\infty} \in \ell_{\tilde{w}_{2}, m_{0}}^{q} .
\end{aligned}
$$

Proof Applying Hölder's inequality, we observe

$$
\begin{aligned}
& \sum_{m=m_{0}}^{\infty} K_{\alpha, \lambda}(\phi(m), \psi(n)) a_{m} \\
& =\sum_{m=m_{0}}^{\infty}\left(K_{\alpha, \lambda}(\phi(m), \psi(n)) \frac{\phi(m)^{\frac{\alpha+1-\lambda_{2}}{q}}}{\psi(n)^{\frac{\alpha+1-\lambda_{1}}{p}}} \frac{\psi^{\prime}(n)^{\frac{1}{p}}}{\phi^{\prime}(m)^{\frac{1}{q}}} a_{m}\right)\left(\frac{\psi(n)^{\frac{\alpha+1-\lambda_{1}}{p}}}{\phi(m)^{\frac{\alpha+1-\lambda_{2}}{q}}} \frac{\phi^{\prime}(m)^{\frac{1}{q}}}{\psi^{\prime}(n)^{\frac{1}{p}}}\right) \\
& \leq\left(\sum_{m=m_{0}}^{\infty} K_{\alpha, \lambda}(\phi(m), \psi(n)) \frac{\phi(m)^{\left(\alpha+1-\lambda_{2}\right)(p-1)}}{\psi(n)^{\alpha+1-\lambda_{1}}} \frac{\psi^{\prime}(n)}{\phi^{\prime}(m)^{p-1}} a_{m}^{p}\right)^{\frac{1}{p}} \\
& \times\left(\sum_{m=m_{0}}^{\infty} K_{\alpha, \lambda}(\phi(m), \psi(n)) \frac{\psi(n)^{\left(\alpha+1-\lambda_{1}\right)(q-1)}}{\phi(m)^{\alpha+1-\lambda_{2}}} \frac{\phi^{\prime}(m)}{\psi^{\prime}(n)^{q-1}}\right)^{\frac{1}{q}} .
\end{aligned}
$$

By Definition 2.2, we get

$$
\begin{aligned}
& \sum_{m=m_{0}}^{\infty} K_{\alpha, \lambda}(\phi(m), \psi(n)) a_{m} \\
& \quad \leq\left(\int_{0}^{\infty} K_{\alpha, \lambda}(1, t) t^{-1+\lambda_{2}-\alpha} d t\right)^{\frac{1}{q}}\left(\frac{\psi(n)^{q\left(\alpha+1-\lambda_{1}\right)-1}}{\psi^{\prime}(n)^{q-1}}\right)^{\frac{1}{q}} \\
& \quad \times\left(\sum_{m=m_{0}}^{\infty} K_{\alpha, \lambda}(\phi(m), \psi(n)) \frac{\phi(m)^{\left(\alpha+1-\lambda_{2}\right)(p-1)}}{\psi(n)^{\alpha+1-\lambda_{1}}} \frac{\psi^{\prime}(n)}{\phi^{\prime}(m)^{p-1}} a_{m}^{p}\right)^{\frac{1}{p}} .
\end{aligned}
$$

Therefore, by using Lemma 2.5, we get

$$
\begin{gathered}
\left\|\sum_{m=m_{0}}^{\infty} K_{\alpha, \lambda}(\phi(m), \psi(n)) a_{m}\right\|_{p, \widetilde{w}_{1}} \\
\quad=\left\{\sum_{n=n_{0}}^{\infty} \frac{\psi^{\prime}(n)}{\psi(n)^{p\left(\alpha-\lambda_{1}\right)+1}}\left(\sum_{m=m_{0}}^{\infty} K_{\alpha, \lambda}(\phi(m), \psi(n)) a_{m}\right)^{p}\right\}^{\frac{1}{p}}
\end{gathered}
$$




$$
\begin{aligned}
& \leq K_{\alpha}(\lambda)^{\frac{1}{q}}\left(\sum_{n=n_{0}}^{\infty} \sum_{m=m_{0}}^{\infty} K_{\alpha, \lambda}(\phi(m), \psi(n)) \frac{\phi(m)^{\left(\alpha+1-\lambda_{2}\right)(p-1)}}{\psi(n)^{\alpha+1-\lambda_{1}}} \frac{\psi^{\prime}(n)}{\phi^{\prime}(m)^{p-1}} a_{m}^{p}\right)^{\frac{1}{p}} \\
& =K_{\alpha}(\lambda)^{\frac{1}{q}}\left(\sum_{m=m_{0}}^{\infty} W_{1}(m) \frac{\phi(m)^{p\left(\alpha+1-\lambda_{2}\right)-1}}{\phi^{\prime}(m)^{p-1}} a_{m}^{p}\right)^{\frac{1}{p}} \\
& <K_{\alpha}(\lambda)\|a\|_{p, w_{1}} .
\end{aligned}
$$

In the same manner, one can obtain

$$
\left\|\sum_{n=n_{0}}^{\infty} K_{\alpha, \lambda}(\phi(m), \psi(n)) b_{n}\right\|_{q, \widetilde{w}_{2}} \leq K_{\alpha}(\lambda)\|b\|_{q, w_{2}}
$$

In view of Lemma 2.6, we can define a Hilbert-type operator $T: \ell_{w_{1}, m_{0}}^{p} \rightarrow \ell_{\widetilde{w}_{1}, n_{0}}^{p}$ by

$$
(T a)(n):=\sum_{m=m_{0}}^{\infty} K_{\alpha, \lambda}(\phi(m), \psi(n)) a_{m}, \quad n \geq n_{0}, n \in \mathbb{Z}
$$

Similarly, define $T: \ell_{w_{2}, n_{0}}^{q} \rightarrow \ell_{\widetilde{w}_{2}, m_{0}}^{q}$ by

$$
(T a)(m):=\sum_{n=n_{0}}^{\infty} K_{\alpha, \lambda}(\phi(m), \psi(n)) b_{n}, \quad m \geq m_{0}, m \in \mathbb{Z}
$$

It immediately follows from Lemma 2.6 that

$$
\|T\|_{p}:=\sup _{\|a\|_{p, \widetilde{w}_{1}=1}}\|T a\|_{p, \widetilde{w}_{1}} \leq K_{\alpha}(\lambda)
$$

and

$$
\|T\|_{q}:=\sup _{\|a\|_{p, \widetilde{w}_{2}=1}}\|T b\|_{q, \widetilde{w}_{2}} \leq K_{\alpha}(\lambda)
$$

Hence the operator $T$ is bounded. The formal inner product $(T a, b)$ of $T a$ and $b$ is defined by

$$
(T a, b):=\sum_{n=n_{0}}^{\infty} \sum_{m=m_{0}}^{\infty} K_{\alpha, \lambda}(\phi(m), \psi(n)) a_{m} b_{n} .
$$

Lemma 2.7 Let $p>1, \frac{1}{p}+\frac{1}{q}=1$. Let $\widetilde{a}=\left\{\widetilde{a}_{m}\right\}_{m=m_{0}}^{\infty}$ and $\widetilde{b}=\left\{\widetilde{b}_{n}\right\}_{n=n_{0}}^{\infty}$ with $\widetilde{a}_{m}=\frac{\phi^{\prime}(m)}{\phi(m)^{\alpha+1-\lambda_{2}+\frac{\varepsilon}{p}}}$ and $\widetilde{b}_{n}=\frac{\psi^{\prime}(n)}{\psi(n)^{\alpha+1-\lambda_{1}+\frac{\varepsilon}{q}}}$ for $0<\varepsilon<p \lambda_{i}, i=1,2$. Then, as $\varepsilon \rightarrow 0+$,

$$
K_{\alpha}(\lambda)(1-o(1)) \sum_{n=n_{0}}^{\infty} \frac{\psi^{\prime}(n)}{\psi(n)^{1+\varepsilon}}<(T \widetilde{a}, \widetilde{b})<K_{\alpha}(\lambda)(1+o(1)) \sum_{n=n_{0}}^{\infty} \frac{\psi^{\prime}(n)}{\psi(n)^{1+\varepsilon}}
$$


Proof We have

$$
\begin{aligned}
(T \widetilde{a}, \tilde{b}) & =\sum_{n=n_{0}}^{\infty} \sum_{m=m_{0}}^{\infty} K_{\alpha, \lambda}(\phi(m), \psi(n)) \frac{\phi^{\prime}(m)}{\left.\phi^{(} m\right)^{\alpha+1-\lambda_{2}+\frac{\varepsilon}{p}}} \frac{\psi^{\prime}(n)}{\psi(n)^{\alpha+1-\lambda_{1}+\frac{\varepsilon}{q}}} \\
& <\sum_{n=n_{0}}^{\infty} \int_{m_{0}-1}^{\infty} K_{\alpha, \lambda}(\phi(x), \psi(n)) \frac{\phi^{\prime}(x)}{\phi(x)^{\alpha+1-\lambda_{2}+\frac{\varepsilon}{p}}} \frac{\psi^{\prime}(n)}{\psi(n)^{\alpha+1-\lambda_{1}+\frac{\varepsilon}{q}}} d x .
\end{aligned}
$$

Setting $t=\frac{\phi(x)}{\psi(n)}$, we get

$$
\begin{aligned}
(T \tilde{a}, \tilde{b}) & <\sum_{n=n_{0}}^{\infty}\left(\int_{0}^{\infty} K_{\alpha, \lambda}(1, t) t^{-1+\lambda_{2}-\alpha-\frac{\varepsilon}{p}} d t\right) \frac{\psi^{\prime}(n)}{\psi(n)^{1+\varepsilon}} \\
& =K_{\alpha}(\lambda)(1+o(1)) \sum_{n=n_{0}}^{\infty} \frac{\psi^{\prime}(n)}{\psi(n)^{1+\varepsilon}} .
\end{aligned}
$$

Moreover,

$$
\begin{aligned}
(T \widetilde{a}, \tilde{b}) & >\sum_{n=n_{0}}^{\infty}\left(\int_{\frac{\phi\left(m_{0}\right)}{\psi(n)}}^{\infty} K_{\alpha, \lambda}(1, t) t^{-1+\lambda_{2}-\alpha-\frac{\varepsilon}{p}} d t\right) \frac{\psi^{\prime}(n)}{\psi(n)^{1+\varepsilon}} \\
& =\sum_{n=n_{0}}^{\infty} \frac{\psi^{\prime}(n)}{\psi(n)^{1+\varepsilon}}\left(\int_{0}^{\infty} K_{\alpha, \lambda}(1, t) t^{-1+\lambda_{2}-\alpha-\frac{\varepsilon}{p}} d t-\int_{0}^{\frac{\phi\left(m_{0}\right)}{\psi(n)}} K_{\alpha, \lambda}(1, t) t^{-1+\lambda_{2}-\alpha-\frac{\varepsilon}{p}} d t\right) .
\end{aligned}
$$

Note that the definition of $K_{\alpha, \lambda}(x, y)$ implies that

$$
\int_{0}^{\infty} K_{\alpha, \lambda}(1, t) t^{-1+\lambda_{2}-\alpha-\frac{\varepsilon}{p}} d t=K_{\alpha}\left(\lambda_{2}\right)+o(1)
$$

and

$$
\sum_{n=n_{0}}^{\infty} \frac{\psi^{\prime}(n)}{\psi(n)^{1+\varepsilon}} \int_{0}^{\frac{\phi\left(m_{0}\right)}{\psi(n)}} K_{\alpha, \lambda}(1, t) t^{-1+\lambda_{2}-\alpha-\frac{\varepsilon}{p}} d t=O(1) .
$$

Thus, using the fact that for $a>0$,

$$
\sum_{n=n_{0}}^{\infty} \frac{\psi^{\prime}(n)}{\psi(n)^{1+\varepsilon}}=\frac{1}{\varepsilon}(1+o(1)) \quad \text { and } \quad \sum_{n=n_{0}}^{\infty} \frac{\psi^{\prime}(n)}{\psi(n)^{1+a+\frac{\varepsilon}{q}}}=O(1)
$$

as $\varepsilon \rightarrow 0+$, we obtain

$$
\begin{aligned}
(T \widetilde{a}, \tilde{b}) & >K_{\alpha}(\lambda)(1+o(1))\left(\sum_{n=n_{0}}^{\infty} \frac{\psi^{\prime}(n)}{\psi(n)^{1+\varepsilon}}-O(1)\right) \\
& =K_{\alpha}(\lambda)\left[1+o(1)-O(1) \sum_{n=n_{0}}^{\infty}\left(\frac{\psi^{\prime}(n)}{\psi(n)^{1+\varepsilon}}\right)^{-1}\right] \sum_{n=n_{0}}^{\infty} \frac{\psi^{\prime}(n)}{\psi(n)^{1+\varepsilon}} \\
& =K_{\alpha}(\lambda)(1-o(1)) \sum_{n=n_{0}}^{\infty} \frac{\psi^{\prime}(n)}{\psi(n)^{1+\varepsilon}},
\end{aligned}
$$

which completes the proof. 
Theorem 2.8 Let $p>1, \frac{1}{p}+\frac{1}{q}=1, \lambda_{1}+\lambda_{2}=\lambda, \lambda_{1}, \lambda_{2}>0$. For $a_{m}, b_{n} \geq 0\left(m_{0}, n_{0} \in \mathbb{Z}\right)$, let $a=\left\{a_{m}\right\}_{m=m_{0}}^{\infty} \in \ell_{w_{1}, m_{0}}^{p}$ and $b=\left\{b_{n}\right\}_{n=n_{0}}^{\infty} \in \ell_{w_{2}, n_{0}}^{q}$. Then, for $\phi(x) \in F_{m_{0}}(r)$ and $\psi(y) \in F_{n_{0}}(s)$ $(r, s>1)$,

$$
\|T\|_{p}=\|T\|_{q}=K_{\alpha}(\lambda)
$$

Proof Suppose that $\|T\|_{p}<K_{\alpha}(\lambda)$. Consider $\widetilde{a}_{m}=\phi^{\prime}(m) \phi(m)^{-1+\lambda_{2}-\alpha-\frac{\varepsilon}{p}}$ and $\widetilde{b}_{n}=\phi^{\prime}(n) \times$ $\psi(n)^{-1+\lambda_{1}-\alpha-\frac{\varepsilon}{q}}$, where $m \geq m_{0}, n \geq n_{0}, m, n \in \mathbb{Z}, 0<\varepsilon<p \lambda_{i}, i=1$, 2. A simple computation shows that $\tilde{a} \in \ell_{w_{1}, m_{0}}^{p}$ and $\tilde{b} \in \ell_{w_{2}, n_{0}}^{q}$ with $\|\tilde{a}\|_{p, w_{1}}>0$ and $\|\tilde{b}\|_{q, w_{2}}>0$. Then

$$
\begin{aligned}
\|T \widetilde{a}\|_{p, \widetilde{w}_{1}} & =\left\{\sum_{n=n_{0}}^{\infty} \psi^{\prime}(n) \psi(n)^{p\left(\lambda_{1}-\alpha\right)-1}\left(\sum_{m=m_{0}}^{\infty} K_{\alpha, \lambda}(\phi(m), \psi(n)) \widetilde{a}_{m}\right)^{p}\right\}^{\frac{1}{p}} \\
& \leq\|T\|_{p}\|\widetilde{a}\|_{p, w_{1}} .
\end{aligned}
$$

Moreover, we have

$$
\begin{aligned}
(T \widetilde{a}, \widetilde{b}) & =\sum_{n=n_{0}}^{\infty} \sum_{m=m_{0}}^{\infty} K_{\alpha, \lambda}(\phi(m), \psi(n)) \widetilde{a}_{m} \widetilde{b}_{n} \\
& =\sum_{n=n_{0}}^{\infty}\left\{\psi^{\prime}(n) \psi(n)^{p\left(\lambda_{1}-\alpha\right)-1}\left(\sum_{m=m_{0}}^{\infty} K_{\alpha, \lambda}(\phi(m), \psi(n)) \tilde{a}_{m}\right)^{p}\right\}^{\frac{1}{p}}\|\widetilde{b}\|_{q, w_{2}} \\
& \leq\|T\|_{p}\|\tilde{a}\|_{p, w_{1}}\|\tilde{b}\|_{q, w_{2}} \\
& =\|T\|_{p}\left(\sum_{m=m_{0}}^{\infty} \frac{\phi^{\prime}(m)}{\phi(m)^{1+\varepsilon}}\right)^{\frac{1}{p}}\left(\sum_{n=n_{0}}^{\infty} \frac{\psi^{\prime}(n)}{\psi(n)^{1+\varepsilon}}\right)^{\frac{1}{q}} .
\end{aligned}
$$

On the other hand, from Lemma 2.7 it follows

$$
K_{\alpha}(\lambda)(1-o(1)) \sum_{n=n_{0}}^{\infty} \frac{\psi^{\prime}(n)}{\psi(n)^{1+\varepsilon}}<(T \widetilde{a}, \tilde{b})
$$

Therefore, combining these inequalities (2) and (3),

$$
K_{\alpha}(\lambda)(1-o(1))\left(\sum_{n=n_{0}}^{\infty} \frac{\psi^{\prime}(n)}{\psi(n)^{1+\varepsilon}}\right)^{\frac{1}{p}} \leq\|T\|_{p}\left(\sum_{m=m_{0}}^{\infty} \frac{\phi^{\prime}(m)}{\phi(m)^{1+\varepsilon}}\right)^{\frac{1}{p}}
$$

Since

$$
\sum_{n=n_{0}}^{\infty} \frac{\psi^{\prime}(n)}{\psi(n)^{1+\varepsilon}}=\frac{1}{\varepsilon}(1+o(1)) \quad \text { and } \quad \sum_{m=m_{0}}^{\infty} \frac{\phi^{\prime}(m)}{\phi(m)^{1+\varepsilon}}=\frac{1}{\varepsilon}(1+o(1))
$$

as $\varepsilon \rightarrow 0+$, we obtain that $K_{\alpha}(\lambda) \leq\|T\|_{p}$, which is a contradiction. Thus we conclude that $\|T\|_{p}=K_{\alpha}(\lambda)$. Applying the same argument, we have $\|T\|_{q}=K_{\alpha}(\lambda)$, which completes the proof. 


\section{Two equivalent inequalities for the Hilbert-type operator}

Equipped with the Hilbert-type operator defined as above, we have the following theorem.

Theorem 3.1 Let $p>1, \frac{1}{p}+\frac{1}{q}=1, \lambda_{1}+\lambda_{2}=\lambda, \lambda_{1}, \lambda_{2}>0$. For $a_{m}, b_{n} \geq 0\left(m_{0}, n_{0} \in \mathbb{Z}\right)$, let $a=\left\{a_{m}\right\}_{m=m_{0}}^{\infty} \in \ell_{w_{1}, m_{0}}^{p}, b=\left\{b_{n}\right\}_{n=n_{0}}^{\infty} \in \ell_{w_{2}, n_{0}}^{q}\|a\|_{p, w_{1}}>0,\|b\|_{q, w_{2}}>0$. Then, for $\phi(x) \in F_{m_{0}}(r)$ and $\psi(y) \in F_{n_{0}}(s)(r, s>1)$, we have the following equivalent inequalities:

$$
\begin{aligned}
& (T a, b)=\sum_{n=n_{0}}^{\infty} \sum_{m=m_{0}}^{\infty} K_{\alpha, \lambda}(\phi(m), \psi(n)) a_{m} b_{n}<K_{\alpha}(\lambda)\|a\|_{p, w_{1}}\|b\|_{q, w_{2}}, \\
& \|T a\|_{p, \widetilde{w}_{1}}<K_{\alpha}(\lambda)\|a\|_{p, w_{1}} .
\end{aligned}
$$

Furthermore, the constant factor $K_{\alpha}(\lambda)$ is the best possible.

Proof It follows from Hölder's inequality that

$$
\begin{aligned}
(T a, b)= & \sum_{n=n_{0}}^{\infty} \sum_{m=m_{0}}^{\infty} K_{\alpha, \lambda}(\phi(m), \psi(n))\left(\frac{\phi(m)^{\frac{\alpha+1-\lambda_{2}}{q}}}{\psi(n)^{\frac{\alpha+1-\lambda_{1}}{p}}} \frac{\psi^{\prime}(n)^{\frac{1}{p}}}{\phi^{\prime}(m)^{\frac{1}{q}}} a_{m}\right) \\
& \times\left(\frac{\psi(n)^{\frac{\alpha+1-\lambda_{1}}{p}}}{\phi(m)^{\frac{\alpha+1-\lambda_{2}}{q}}} \frac{\phi^{\prime}(m)^{\frac{1}{q}}}{\psi^{\prime}(n)^{\frac{1}{p}}} b_{n}\right) \\
\leq & \left(\sum_{n=n_{0}}^{\infty} \sum_{m=m_{0}}^{\infty} K_{\alpha, \lambda}(\phi(m), \psi(n)) \frac{\phi(m)^{\left(\alpha+1-\lambda_{2}\right)(p-1)}}{\psi(n)^{\alpha+1-\lambda_{1}}} \frac{\psi^{\prime}(n)}{\phi^{\prime}(m)^{p-1}} a_{m}^{p}\right)^{\frac{1}{p}} \\
& \times\left(\sum_{n=n_{0}}^{\infty} \sum_{m=m_{0}}^{\infty} K_{\alpha, \lambda}(\phi(m), \psi(n)) \frac{\psi(n)^{\left(\alpha+1-\lambda_{1}\right)(q-1)}}{\phi(m)^{\alpha+1-\lambda_{2}}} \frac{\phi^{\prime}(m)}{\psi^{\prime}(n)^{q-1}} b_{n}^{q}\right)^{\frac{1}{q}} \\
= & \left(\sum_{m=m_{0}}^{\infty} W_{1}(m) \frac{\phi(m)^{p\left(\alpha+1-\lambda_{2}\right)-1}}{\phi^{\prime}(m)^{p-1}} a_{m}^{p}\right)^{\frac{1}{p}}\left(\sum_{n=n_{0}}^{\infty} W_{2}(n) \frac{\psi(n)^{q\left(\alpha+1-\lambda_{1}\right)-1}}{\psi^{\prime}(n)^{q-1}} b_{n}^{q}\right)^{\frac{1}{q}} .
\end{aligned}
$$

Applying Lemma 2.5, we see that

$$
(T a, b)<K_{\alpha}(\lambda)\|a\|_{p, w_{1}}\|b\|_{q, w_{2}} .
$$

In order to prove that inequality (4) implies inequality (5), we define $\widetilde{b}$ as follows:

$$
\widetilde{b}_{n}:=\frac{\psi^{\prime}(n)}{\psi(n)^{p\left(\alpha-\lambda_{1}\right)+1}}\left(\sum_{m=m_{0}}^{\infty} K_{\alpha, \lambda}(\phi(m), \psi(n))\right)^{p-1}
$$

for $n \geq n_{0}, n \in \mathbb{Z}$. Then we see that $\widetilde{b} \in \ell_{w_{2}, n_{0}}^{q}$ and $\|\widetilde{b}\|_{q, w_{2}}>0$ as before. Thus using inequality (4) shows that

$$
\begin{aligned}
\|\widetilde{b}\|_{q, w_{2}}^{q} & =\sum_{n=n_{0}}^{\infty} \frac{\psi^{\prime}(n)}{\psi(n)^{p\left(\alpha-\lambda_{1}\right)+1}}\left(\sum_{m=m_{0}}^{\infty} K_{\alpha, \lambda}(\phi(m), \psi(n)) a_{m}\right)^{p} \\
& =\sum_{n=n_{0}}^{\infty} \sum_{m=m_{0}}^{\infty} K_{\alpha, \lambda}(\phi(m), \psi(n)) a_{m} \widetilde{b}_{n}<K_{\alpha}(\lambda)\|a\|_{p, w_{1}}\|\widetilde{b}\|_{q, w_{2}},
\end{aligned}
$$


which gives $\|T a\|_{p, \widetilde{w}_{1}}=\|\widetilde{b}\|_{q, w_{2}}^{q-1}<K_{\alpha}(\lambda)\|a\|_{p, w_{1}}$. Hence inequality (4) implies inequality (5).

Now suppose that inequality (5) holds for any $a \in \ell_{w_{1}, m_{0}}^{p}$.

$$
\begin{aligned}
(T a, b) & =\sum_{n=n_{0}}^{\infty} \sum_{m=m_{0}}^{\infty} K_{\alpha, \lambda}(\phi(m), \psi(n)) a_{m} b_{n} \\
& =\sum_{n=n_{0}}^{\infty}\left(\frac{\psi^{\prime}(n)^{\frac{1}{p}}}{\psi(n)^{\alpha-\lambda_{1}+\frac{1}{p}}} \sum_{m=m_{0}}^{\infty} K_{\alpha, \lambda}(\phi(m), \psi(n)) a_{m}\right)\left(\frac{\psi(n)^{\alpha-\lambda_{1}+\frac{1}{p}}}{\psi^{\prime}(n)^{\frac{1}{p}}} b_{n}\right) \\
& \leq\left\{\sum_{n=n_{0}}^{\infty} \frac{\psi^{\prime}(n)}{\psi(n)^{p\left(\alpha-\lambda_{1}\right)+1}}\left(\sum_{m=m_{0}}^{\infty} K_{\alpha, \lambda}(\phi(m), \psi(n)) a_{m}\right)^{p}\right\}^{\frac{1}{p}}\|b\|_{q, w_{2}} \\
& <K_{\alpha}(\lambda)\|a\|_{p, w_{1}}\|b\|_{q, w_{2}},
\end{aligned}
$$

which means that inequality (5) implies inequality (4). Therefore inequality (4) is equivalent to inequality (5). Furthermore, Theorem 2.8 implies that the constant factor $K_{\alpha}(\lambda)$ in inequalities (4) and (5) is the best possible, which completes the proof.

\section{Applications to various Hilbert-type inequalities}

In this section, we apply our previous theorems to obtain several Hilbert-type inequalities.

Recall that the beta function $B(u, v)$ is defined by

$$
B(u, v):=\int_{0}^{\infty} \frac{t^{u-1}}{(1+t)^{u+v}} d t=B(u, v) \quad(u, v>0) .
$$

Define the function $K_{\alpha, \lambda}(x, y)$ by

$$
K_{\alpha, \lambda}(x, y):=\frac{(x y)^{\alpha}}{(x+y)^{\lambda}}
$$

for $\lambda>\alpha \geq 0$. Then $K_{\alpha, \lambda}(x, y)$ is a symmetric homogeneous function of degree $2 \alpha-\lambda$ and is decreasing with respect to $x$ and $y$, respectively. Moreover,

$$
\sum_{n=n_{0}}^{\infty} \frac{\psi^{\prime}(n)}{\psi(n)^{1+\varepsilon}} \int_{0}^{\frac{\phi\left(m_{0}\right)}{\psi(n)}} K_{\alpha, \lambda}(1, t) t^{-1+\lambda_{2}-\alpha-\frac{\varepsilon}{p}} d t=O(1)
$$

To see this, for $0<\varepsilon<p \lambda_{2}$,

$$
\begin{aligned}
\sum_{n=n_{0}}^{\infty} \frac{\psi^{\prime}(n)}{\psi(n)^{1+\varepsilon}} \int_{0}^{\frac{\phi\left(m_{0}\right)}{\psi(n)}} \frac{t^{-1+\lambda_{2}-\frac{\varepsilon}{p}}}{(1+t)^{\lambda}} d t & \leq \sum_{n=n_{0}}^{\infty} \frac{\psi^{\prime}(n)}{\psi(n)^{1+\varepsilon}} \int_{0}^{\frac{\phi\left(m_{0}\right)}{\psi(n)}} t^{-1+\lambda_{2}-\frac{\varepsilon}{p}} d t \\
& =\sum_{n=n_{0}}^{\infty} \frac{\psi^{\prime}(n)}{\psi(n)^{1+\varepsilon}} \frac{1}{\lambda_{2}-\frac{\varepsilon}{p}}\left(\frac{\phi\left(m_{0}\right)}{\psi(n)}\right)^{\lambda_{2}-\frac{\varepsilon}{p}} \\
& =\frac{\phi\left(m_{0}\right)^{\lambda_{2}-\frac{\varepsilon}{p}}}{\lambda_{2}-\frac{\varepsilon}{p}} \sum_{n=n_{0}}^{\infty} \frac{\psi^{\prime}(n)}{\psi(n)^{1+\lambda_{2}+\frac{\varepsilon}{q}}} \\
& =O(1) .
\end{aligned}
$$


Note that since

$$
\begin{aligned}
\widetilde{K}_{\alpha, \lambda}\left(\lambda_{i}, \varepsilon\right) & :=\int_{0}^{\infty} K_{\alpha, \lambda}(1, t) t^{-1+\lambda_{i}-\alpha-\varepsilon} d t \\
& =\int_{0}^{\infty} \frac{t^{-1+\lambda_{i}-\varepsilon}}{(1+t)^{\lambda}} d t
\end{aligned}
$$

we see that

$$
\widetilde{K}_{\alpha, \lambda}\left(\lambda_{i}, \varepsilon\right) \rightarrow \int_{0}^{\infty} \frac{t^{\lambda_{i}-1}}{(1+t)^{\lambda}} d t=B\left(\lambda_{1}, \lambda_{2}\right)=K_{\alpha}\left(\lambda_{i}\right)=K_{\alpha}(\lambda)
$$

as $\varepsilon \rightarrow 0+$. Therefore from Theorem 3.1 we observe the following.

Corollary 4.1 Let $p>1, \frac{1}{p}+\frac{1}{q}=1, \lambda_{1}+\lambda_{2}=\lambda, \lambda_{1}, \lambda_{2}>0, \lambda>\alpha \geq 0$. For $a_{m}, b_{n} \geq 0$ $\left(m_{0}, n_{0} \in \mathbb{Z}\right)$, let $a=\left\{a_{m}\right\}_{m=m_{0}}^{\infty} \in \ell_{w_{1}, m_{0}}^{p}, b=\left\{b_{n}\right\}_{n=n_{0}}^{\infty} \in \ell_{w_{2}, n_{0}}^{q}$ and $\|a\|_{p, w_{1}}>0,\|b\|_{q, w_{2}}>0$. Then, for $\phi(x) \in F_{m_{0}}(r)$ and $\psi(y) \in F_{n_{0}}(s)(r, s>1)$, we have the following equivalent inequalities:

$$
\begin{aligned}
& \sum_{n=n_{0}}^{\infty} \sum_{m=m_{0}}^{\infty} \frac{\phi(m)^{\alpha} \psi(n)^{\alpha} a_{m} b_{n}}{(\phi(m)+\psi(n))^{\lambda}}<B\left(\lambda_{1}, \lambda_{2}\right)\|a\|_{p, w_{1}}\|b\|_{q, w_{2}}, \\
& \left\{\sum_{n=n_{0}}^{\infty} \psi^{\prime}(n) \psi(n)^{p\left(\lambda_{1}-\alpha\right)-1}\left(\sum_{m=m_{0}}^{\infty} \frac{\phi(m)^{\alpha} \psi(n)^{\alpha} a_{m}}{(\phi(m)+\psi(n))^{\lambda}}\right)^{p}\right\}^{\frac{1}{p}}<B\left(\lambda_{1}, \lambda_{2}\right)\|a\|_{p, w_{1}} .
\end{aligned}
$$

Furthermore, the constant factor $B\left(\lambda_{1}, \lambda_{2}\right)$ is the best possible.

As applications, we have the following.

Case 1. Let $\phi(x)=x^{\beta}$ and $\psi(x)=x^{\gamma}(\beta, \gamma>0)$ for $m_{0}=n_{0}=1$. For $0<\lambda_{i}<\alpha+\min \left\{\frac{1}{\beta}, \frac{1}{\gamma}\right\}$ and $0 \leq \alpha<\lambda$, one has the following equivalent inequalities:

$$
\begin{aligned}
& \sum_{n=1}^{\infty} \sum_{m=1}^{\infty} \frac{\left(m^{\beta} n^{\gamma}\right)^{\alpha}}{\left(m^{\beta}+n^{\gamma}\right)^{\lambda}} a_{m} b_{n}<\frac{B\left(\lambda_{1}, \lambda_{2}\right)}{\beta^{\frac{1}{q}} \gamma^{\frac{1}{p}}}\|a\|_{p, w_{1}}\|b\|_{q, w_{2}}, \\
& \left\{\sum_{n=1}^{\infty} n^{\gamma p\left(\lambda_{1}-\alpha\right)-1}\left(\sum_{m=1}^{\infty} \frac{\left(m^{\beta} n^{\gamma}\right)^{\alpha}}{\left(m^{\beta}+n^{\gamma}\right)^{\lambda}} a_{m}\right)^{p}\right\}^{\frac{1}{p}}<\frac{B\left(\lambda_{1}, \lambda_{2}\right)}{\beta^{\frac{1}{q}} \gamma^{\frac{1}{p}}}\|a\|_{p, w_{1}},
\end{aligned}
$$

where $w_{1}(m)=m^{p\left(1-\lambda_{2} \beta+\alpha \beta\right)-1}$ and $w_{2}(n)=n^{q\left(1-\lambda_{1} \gamma+\alpha \gamma\right)-1}$.

(I) For $\lambda_{1}=\frac{\lambda}{p}$ and $\lambda_{2}=\frac{\lambda}{q}$ with $0<\lambda_{i}<\alpha+\min \left\{\frac{1}{\beta}, \frac{1}{\gamma}\right\}$ and $0 \leq \alpha<\lambda$, one has the following equivalent inequalities:

$$
\begin{aligned}
& \sum_{n=1}^{\infty} \sum_{m=1}^{\infty} \frac{\left(m^{\beta} n^{\gamma}\right)^{\alpha}}{\left(m^{\beta}+n^{\gamma}\right)^{\lambda}} a_{m} b_{n}<\frac{B\left(\frac{\lambda}{p}, \frac{\lambda}{q}\right)}{\beta^{\frac{1}{q}} \gamma^{\frac{1}{p}}}\|a\|_{p, w_{1}}\|b\|_{q, w_{2}}, \\
& \left\{\sum_{n=1}^{\infty} n^{\gamma(\lambda-p \alpha)-1}\left(\sum_{m=1}^{\infty} \frac{\left(m^{\beta} n^{\gamma}\right)^{\alpha}}{\left(m^{\beta}+n^{\gamma}\right)^{\lambda}} a_{m}\right)^{p}\right\}^{\frac{1}{p}}<\frac{B\left(\frac{\lambda}{p}, \frac{\lambda}{q}\right)}{\beta^{\frac{1}{q}} \gamma^{\frac{1}{p}}}\|a\|_{p, w_{1}},
\end{aligned}
$$

where $w_{1}(m)=m^{(p-1)(1-\lambda \beta)+p \alpha \beta}$ and $w_{2}(n)=n^{(q-1)(1-\lambda \gamma)+q \alpha \gamma}$. 
(II) For $\lambda_{1}=\frac{\lambda}{q}$ and $\lambda_{2}=\frac{\lambda}{p}$ with $0<\lambda_{i}<\alpha+\min \left\{\frac{1}{\beta}, \frac{1}{\gamma}\right\}$ and $0 \leq \alpha<\lambda$, one has the following equivalent inequalities:

$$
\begin{aligned}
& \sum_{n=1}^{\infty} \sum_{m=1}^{\infty} \frac{\left(m^{\beta} n^{\gamma}\right)^{\alpha}}{\left(m^{\beta}+n^{\gamma}\right)^{\lambda}} a_{m} b_{n}<\frac{B\left(\frac{\lambda}{p}, \frac{\lambda}{q}\right)}{\beta^{\frac{1}{q}} \gamma^{\frac{1}{p}}}\|a\|_{p, w_{1}}\|b\|_{q, w_{2}}, \\
& \left\{\sum_{n=1}^{\infty} n^{\gamma \lambda(p-1)-p \alpha \gamma-1}\left(\sum_{m=1}^{\infty} \frac{\left(m^{\beta} n^{\gamma}\right)^{\alpha}}{\left(m^{\beta}+n^{\gamma}\right)^{\lambda}} a_{m}\right)^{p}\right\}^{\frac{1}{p}}<\frac{B\left(\frac{\lambda}{p}, \frac{\lambda}{q}\right)}{\beta^{\frac{1}{q}} \gamma^{\frac{1}{p}}}\|a\|_{p, w_{1}},
\end{aligned}
$$

where $w_{1}(m)=m^{p-1-\beta \lambda+p \alpha \beta}$ and $w_{2}(n)=n^{q-1-\gamma \lambda+q \alpha \gamma}$.

(III) Let $\lambda_{1}=\frac{p+\lambda-2}{p}, \lambda_{2}=\frac{q+\lambda-2}{q}, \lambda>\max \{2-p, 2-q\}, 0<\beta<\frac{p}{p+\lambda-2-p \alpha}, 0<\gamma<\frac{q}{q+\lambda-2-q \alpha}$, $0 \leq \alpha<\min \left\{\frac{p+\lambda-2}{p}, \frac{q+\lambda-2}{q}\right\}$. Then one has the following equivalent inequalities:

$$
\begin{aligned}
& \sum_{n=1}^{\infty} \sum_{m=1}^{\infty} \frac{\left(m^{\beta} n^{\gamma}\right)^{\alpha}}{\left(m^{\beta}+n^{\gamma}\right)^{\lambda}} a_{m} b_{n}<\frac{B\left(\frac{p+\lambda-2}{p}, \frac{q+\lambda-2}{q}\right)}{\beta^{\frac{1}{q}} \gamma^{\frac{1}{p}}}\|a\|_{p, w_{1}}\|b\|_{q, w_{2}}, \\
& \left\{\sum_{n=1}^{\infty} n^{\gamma(p+\lambda-2)-p \alpha \gamma-1}\left(\sum_{m=1}^{\infty} \frac{\left(m^{\beta} n^{\gamma}\right)^{\alpha}}{\left(m^{\beta}+n^{\gamma}\right)^{\lambda}} a_{m}\right)^{p}\right\}^{\frac{1}{p}}<\frac{B\left(\frac{p+\lambda-2}{p}, \frac{q+\lambda-2}{q}\right)}{\beta^{\frac{1}{q}} \gamma^{\frac{1}{p}}}\|a\|_{p, w_{1}},
\end{aligned}
$$

where $w_{1}(m)=m^{(p-1)(1-\beta(q+\lambda-2))+p \alpha \beta}$ and $w_{2}(n)=n^{(q-1)(1-\gamma(p+\lambda-2))+q \alpha \gamma}$.

(IV) Let $\lambda_{1}=\frac{q+\lambda-2}{q}, \lambda_{2}=\frac{p+\lambda-2}{p}, \lambda>\max \{2-p, 2-q\}, 0<\beta<\frac{q}{q+\lambda-2-q \alpha}, 0<\gamma<\frac{p}{p+\lambda-2-p \alpha}$, $0 \leq \alpha<\min \left\{\frac{p+\lambda-2}{p}, \frac{q+\lambda-2}{q}\right\}$. Then one has the following equivalent inequalities:

$$
\begin{aligned}
& \sum_{n=1}^{\infty} \sum_{m=1}^{\infty} \frac{\left(m^{\beta} n^{\gamma}\right)^{\alpha}}{\left(m^{\beta}+n^{\gamma}\right)^{\lambda}} a_{m} b_{n}<\frac{B\left(\frac{p+\lambda-2}{p}, \frac{q+\lambda-2}{q}\right)}{\beta^{\frac{1}{q}} \gamma^{\frac{1}{p}}}\|a\|_{p, w_{1}}\|b\|_{q, w_{2}}, \\
& \left\{\sum_{n=1}^{\infty} n^{\gamma(p-1)(q+\lambda-2)-p \alpha \gamma-1}\left(\sum_{m=1}^{\infty} \frac{\left(m^{\beta} n^{\gamma}\right)^{\alpha}}{\left(m^{\beta}+n^{\gamma}\right)^{\lambda}} a_{m}\right)^{p}\right\}^{\frac{1}{p}}<\frac{B\left(\frac{p+\lambda-2}{p}, \frac{q+\lambda-2}{q}\right)}{\beta^{\frac{1}{q}} \gamma^{\frac{1}{p}}}\|a\|_{p, w_{1}},
\end{aligned}
$$

where $w_{1}(m)=m^{p-1-\beta(p+\lambda-2)+p \alpha \beta}$ and $w_{2}(n)=n^{q-1-\gamma(q+\lambda-2)+q \alpha \gamma}$.

Case 2. For $A, B>0$, let $\phi(x)=A(\ln x)^{\beta}$ and $\psi(x)=B(\ln x)^{\gamma}(\beta, \gamma>0), m_{0}=n_{0}=2$. For $0<\lambda_{i}<\alpha+\min \left\{\frac{1}{\beta}, \frac{1}{\gamma}\right\}$ and $0 \leq \alpha<\lambda$, one has the following equivalent inequalities:

$$
\begin{aligned}
& \sum_{n=2}^{\infty} \sum_{m=2}^{\infty} \frac{\left((\ln m)^{\beta}(\ln n)^{\gamma}\right)^{\alpha}}{\left(A(\ln m)^{\beta}+B(\ln n)^{\gamma}\right)^{\lambda}} a_{m} b_{n}<\frac{B\left(\lambda_{1}, \lambda_{2}\right)}{A^{\lambda_{2}} B^{\lambda_{1}} \beta^{\frac{1}{q}} \gamma^{\frac{1}{p}}}\|a\|_{p, w_{1}}\|b\|_{q, w_{2}}, \\
& \left\{\sum_{n=2}^{\infty} \frac{1}{n}(\ln n)^{p \gamma\left(\lambda_{1}-\alpha\right)-1}\left(\sum_{m=2}^{\infty} \frac{\left((\ln m)^{\beta}(\ln n)^{\gamma}\right)^{\alpha}}{\left(A(\ln m)^{\beta}+B(\ln n)^{\gamma}\right)^{\lambda}} a_{m}\right)^{p}\right\}^{\frac{1}{p}}<\frac{B\left(\lambda_{1}, \lambda_{2}\right)}{A^{\lambda_{2}} B^{\lambda_{1}} \beta^{\frac{1}{q}} \gamma^{\frac{1}{p}}}\|a\|_{p, w_{1}},
\end{aligned}
$$

where $w_{1}(m)=m^{p-1}(\ln m)^{p\left(1-\lambda_{2} \beta+\alpha \beta\right)-1}$ and $w_{2}(n)=n^{q-1}(\ln n)^{q\left(1-\lambda_{1} \gamma+\alpha \gamma\right)-1}$.

(I) For $\lambda_{1}=\frac{\lambda}{p}$ and $\lambda_{2}=\frac{\lambda}{q}$ with $0<\lambda_{i}<\alpha+\min \left\{\frac{1}{\beta}, \frac{1}{\gamma}\right\}$ and $0 \leq \alpha<\lambda$, one has the following equivalent inequalities:

$$
\sum_{n=2}^{\infty} \sum_{m=2}^{\infty} \frac{\left((\ln m)^{\beta}(\ln n)^{\gamma}\right)^{\alpha}}{\left(A(\ln m)^{\beta}+B(\ln n)^{\gamma}\right)^{\lambda}} a_{m} b_{n}<\frac{B\left(\frac{\lambda}{p}, \frac{\lambda}{q}\right)}{A^{\lambda_{2}} B^{\lambda_{1}} \beta^{\frac{1}{q}} \gamma^{\frac{1}{p}}}\|a\|_{p, w_{1}}\|b\|_{q, w_{2}}
$$




$$
\begin{aligned}
& \left\{\sum_{n=2}^{\infty} \frac{1}{n}(\ln n)^{\gamma-p \alpha \gamma-1}\left(\sum_{m=2}^{\infty} \frac{\left((\ln m)^{\beta}(\ln n)^{\gamma}\right)^{\alpha}}{\left(A(\ln m)^{\beta}+B(\ln n)^{\gamma}\right)^{\lambda}} a_{m}\right)^{p}\right\}^{\frac{1}{p}} \\
& <\frac{B\left(\frac{\lambda}{p}, \frac{\lambda}{q}\right)}{A^{\lambda_{2}} B^{\lambda_{1}} \beta^{\frac{1}{q}} \gamma^{\frac{1}{p}}}\|a\|_{p, w_{1}}
\end{aligned}
$$

where $w_{1}(m)=m^{p-1}(\ln m)^{(p-1)(1-\lambda \beta)+p \alpha \beta}$ and $w_{2}(n)=n^{q-1}(\ln n)^{(q-1)(1-\lambda \gamma)+q \alpha \gamma}$.

(II) Let $\lambda_{1}=\frac{p+\lambda-2}{p}, \lambda_{2}=\frac{q+\lambda-2}{q}, \lambda>\max \{2-p, 2-q\}, 0<\beta<\frac{p}{p+\lambda-2-p \alpha}, 0<\gamma<\frac{q}{q+\lambda-2-q \alpha}$, $0 \leq \alpha<\min \left\{\frac{p+\lambda-2}{p}, \frac{q+\lambda-2}{q}\right\}$. Then one has the following equivalent inequalities:

$$
\begin{aligned}
& \sum_{n=2}^{\infty} \sum_{m=2}^{\infty} \frac{\left((\ln m)^{\beta}(\ln n)^{\gamma}\right)^{\alpha}}{\left(A(\ln m)^{\beta}+B(\ln n)^{\gamma}\right)^{\lambda}} a_{m} b_{n}<\frac{B\left(\frac{p+\lambda-2}{p}, \frac{q+\lambda-2}{q}\right)}{A^{\lambda_{2}} B^{\lambda_{1}} \beta^{\frac{1}{q}} \gamma^{\frac{1}{p}}}\|a\|_{p, w_{1}}\|b\|_{q, w_{2}}, \\
& \left\{\sum_{n=2}^{\infty} \frac{1}{n}(\ln n)^{\gamma(p+\lambda-2)-p \alpha \gamma-1}\left(\sum_{m=2}^{\infty} \frac{\left((\ln m)^{\beta}(\ln n)^{\gamma}\right)^{\alpha}}{\left(A(\ln m)^{\beta}+B(\ln n)^{\gamma}\right)^{\lambda}} a_{m}\right)^{p}\right\}^{\frac{1}{p}} \\
& <\frac{B\left(\frac{p+\lambda-2}{p}, \frac{q+\lambda-2}{q}\right)}{A^{\lambda_{2}} B^{\lambda_{1}} \beta^{\frac{1}{q}} \gamma^{\frac{1}{p}}}\|a\|_{p, w_{1}},
\end{aligned}
$$

where $w_{1}(m)=m^{p-1}(\ln m)^{(p-1)(1-\beta(q+\lambda-2))+p \alpha \beta}$ and $w_{2}(n)=n^{q-1}(\ln n)^{(q-1)(1-\gamma(p+\lambda-2))+q \alpha \gamma}$.

Case 3. For $A, B>0$, let $\phi(x)=A(\ln x)^{\beta}$ and $\psi(x)=B x^{\gamma}(\beta, \gamma>0), m_{0}=2, n_{0}=1$. For $0<\lambda_{i}<\alpha+\min \left\{\frac{1}{\beta}, \frac{1}{\gamma}\right\}$ and $0 \leq \alpha<\lambda$, one has the following equivalent inequalities:

$$
\begin{aligned}
& \sum_{n=1}^{\infty} \sum_{m=2}^{\infty} \frac{\left((\ln m)^{\beta} n^{\gamma}\right)^{\alpha}}{\left(A(\ln m)^{\beta}+B n^{\gamma}\right)^{\lambda}} a_{m} b_{n}<\frac{B\left(\lambda_{1}, \lambda_{2}\right)}{A^{\lambda_{2}} B^{\lambda_{1}} \beta^{\frac{1}{q}} \gamma^{\frac{1}{p}}}\|a\|_{p, w_{1}}\|b\|_{q, w_{2}}, \\
& \left\{\sum_{n=1}^{\infty} n^{p \gamma\left(\lambda_{1}-\alpha\right)-1}\left(\sum_{m=2}^{\infty} \frac{\left((\ln m)^{\beta} n^{\gamma}\right)^{\alpha}}{\left(A(\ln m)^{\beta}+B n^{\gamma}\right)^{\lambda}} a_{m}\right)^{p}\right\}^{\frac{1}{p}}<\frac{B\left(\lambda_{1}, \lambda_{2}\right)}{A^{\lambda_{2}} B^{\lambda_{1}} \beta^{\frac{1}{q}} \gamma^{\frac{1}{p}}}\|a\|_{p, w_{1}},
\end{aligned}
$$

where $w_{1}(m)=m^{p-1}(\ln m)^{p\left(1-\lambda_{2} \beta+\alpha \beta\right)-1}$ and $w_{2}(n)=n^{q\left(1-\lambda_{1} \gamma+\alpha \gamma\right)-1}$.

(I) For $\lambda_{1}=\frac{\lambda}{p}$ and $\lambda_{2}=\frac{\lambda}{q}$ with $0<\lambda_{i}<\alpha+\min \left\{\frac{1}{\beta}, \frac{1}{\gamma}\right\}$ and $0 \leq \alpha<\lambda$, one has the following equivalent inequalities:

$$
\begin{aligned}
& \sum_{n=1}^{\infty} \sum_{m=2}^{\infty} \frac{\left((\ln m)^{\beta} n^{\gamma}\right)^{\alpha}}{\left(A(\ln m)^{\beta}+B n^{\gamma}\right)^{\lambda}} a_{m} b_{n}<\frac{B\left(\frac{\lambda}{p}, \frac{\lambda}{q}\right)}{A^{\lambda_{2}} B^{\lambda_{1}} \beta^{\frac{1}{q}} \gamma^{\frac{1}{p}}}\|a\|_{p, w_{1}}\|b\|_{q, w_{2}}, \\
& \left\{\sum_{n=1}^{\infty} n^{\gamma(1-p \alpha)-1}\left(\sum_{m=2}^{\infty} \frac{\left((\ln m)^{\beta} n^{\gamma}\right)^{\alpha}}{\left(A(\ln m)^{\beta}+B n^{\gamma}\right)^{\lambda}} a_{m}\right)^{p}\right\}^{\frac{1}{p}}<\frac{B\left(\frac{\lambda}{p}, \frac{\lambda}{q}\right)}{A^{\lambda_{2}} B^{\lambda_{1}} \beta^{\frac{1}{q}} \gamma^{\frac{1}{p}}}\|a\|_{p, w_{1}},
\end{aligned}
$$

where $w_{1}(m)=m^{p-1}(\ln m)^{(p-1)(1-\lambda \beta)+p \alpha \beta}$ and $w_{2}(n)=n^{(q-1)(1-\lambda \gamma)+q \alpha \gamma}$.

(II) Let $\lambda_{1}=\frac{p+\lambda-2}{p}, \lambda_{2}=\frac{q+\lambda-2}{q}, \lambda>\max \{2-p, 2-q\}, 0<\beta<\frac{p}{p+\lambda-2-p \alpha}, 0<\gamma<\frac{q}{q+\lambda-2-q \alpha}$, $0 \leq \alpha<\min \left\{\frac{p+\lambda-2}{p}, \frac{q+\lambda-2}{q}\right\}$. Then one has the following equivalent inequalities:

$$
\sum_{n=1}^{\infty} \sum_{m=2}^{\infty} \frac{\left((\ln m)^{\beta} n^{\gamma}\right)^{\alpha}}{\left(A(\ln m)^{\beta}+B n^{\gamma}\right)^{\lambda}} a_{m} b_{n}<\frac{B\left(\frac{p+\lambda-2}{p}, \frac{q+\lambda-2}{q}\right)}{A^{\lambda_{2}} B^{\lambda_{1}} \beta^{\frac{1}{q}} \gamma^{\frac{1}{p}}}\|a\|_{p, w_{1}}\|b\|_{q, w_{2}}
$$




$$
\left\{\sum_{n=1}^{\infty} n^{\gamma(p+\lambda-2)-p \alpha \gamma-1}\left(\sum_{m=2}^{\infty} \frac{\left((\ln m)^{\beta} n^{\gamma}\right)^{\alpha}}{\left(A(\ln m)^{\beta}+B n^{\gamma}\right)^{\lambda}} a_{m}\right)^{p}\right\}^{\frac{1}{p}}<\frac{B\left(\frac{p+\lambda-2}{p}, \frac{q+\lambda-2}{q}\right)}{A^{\lambda_{2}} B^{\lambda_{1}} \beta^{\frac{1}{q}} \gamma^{\frac{1}{p}}}\|a\|_{p, w_{1}}
$$

where $w_{1}(m)=m^{p-1}(\ln m)^{(p-1)(1-\beta(q+\lambda-2))+p \alpha \beta}$ and $w_{2}(n)=n^{(q-1)(1-\gamma(p+\lambda-2))+q \alpha \gamma}$.

\section{Competing interests}

The author declares that he has no competing interests.

\section{Acknowledgements}

This research was supported by the Sookmyung Women's University Research Grants 2012.

Received: 10 July 2015 Accepted: 17 August 2015 Published online: 02 September 2015

\section{References}

1. Hardy, GH, Littlewood, JE, Polya, G: Inequalities. Cambridge University Press, Cambridge (1952)

2. Azar, LE: On some extensions of Hardy-Hilbert's inequality and applications. J. Inequal. Appl. 2008, Article ID 546829 (2008)

3. Debnath, L, Yang, B: Recent developments of Hilbert-type discrete and integral inequalities with applications. Int. J. Math. Stat. Sci. 2012, Article ID 871845 (2012)

4. Gao, M: A note on the Hardy-Hilbert inequality. J. Math. Anal. Appl. 204(1), 346-351 (1996)

5. Gao, M: On Hilbert's inequality and its applications. J. Math. Anal. Appl. 212(1), 316-323 (1997)

6. Krnić, M, Pečarić, JE: Extension of Hilbert's inequality. J. Math. Anal. Appl. 324(1), 150-160 (2006)

7. Mitrinović, DS, Pečarić, JE: On inequalities of Hilbert and Widder. Proc. Edinb. Math. Soc. 34(3), 411-414 (1991)

8. Pachpatte, BG: Inequalities similar to certain extensions of Hilbert's inequality. J. Math. Anal. Appl. 243(2), 217-227 (2000)

9. Sulaiman, WT: New kinds of Hardy-Hilbert's integral inequalities. Appl. Math. Lett. 23(4), 361-365 (2010)

10. Yang, B: On Hilbert's integral inequality. J. Math. Anal. Appl. 220(2), 778-785 (1998)

11. Yang, B: On new generalizations of Hilbert's inequality. J. Math. Anal. Appl. 248(1), 29-40 (2000)

12. Yang, B: On a relation between Hilbert's inequality and a Hilbert-type inequality. Appl. Math. Lett. 21(5), 483-488 (2008)

13. Yang, B, Debnath, L: Some inequalities involving $\pi$ and an application to Hilbert's inequality. Appl. Math. Lett. 12(8), 101-105 (1999)

14. Yang, B: On the norm of a Hilbert's type linear operator and applications. J. Math. Anal. Appl. 325(1), 529-541 (2007)

15. Jin, J, Debnath, L: On a Hilbert-type linear series operator and its applications. J. Math. Anal. Appl. 371(2), 691-704 (2010)

16. Jichang, K, Debnath, L: On Hilbert type inequalities with non-conjugate parameters. Appl. Math. Lett. 22(5), 813-818 (2009)

17. Yang, B: On a Hilbert-type operator with a symmetric homogeneous kernel of -1-order and applications. J. Inequal. Appl. 2007, Article ID 47812 (2007)

18. Yang, B: On the norm of a self-adjoint operator and a new bilinear integral inequality. Acta Math. Sin. Engl. Ser. 23(7), 1311-1316 (2007)

19. Yang, B: On a Hilbert-type operator with a class of homogeneous kernels. J. Inequal. Appl. 2009. Article ID 572176 (2009)

20. Yang, B: A new Hilbert-type operator and applications. Publ. Math. (Debr.) 76(1-2), 147-156 (2010)

21. Zhong, W: The Hilbert-type integral inequalities with a homogeneous kernel of $-\lambda$-degree. J. Inequal. Appl. 2008 , Article ID 917392 (2008)

22. He, L, Gao, X, Gao, M: On a new weighted Hilbert inequality. J. Inequal. Appl. 2008, Article ID 637397 (2008)

23. Yang, B, Debnath, L: On the extended Hardy-Hilbert's inequality. J. Math. Anal. Appl. 272(1), 187-199 (2002)

\section{Submit your manuscript to a SpringerOpen ${ }^{\circ}$ journal and benefit from:}

- Convenient online submission

Rigorous peer review

- Immediate publication on acceptance

- Open access: articles freely available online

- High visibility within the field

- Retaining the copyright to your article 